CAHIERS DE

NARRATOLOGIE
Cahiers de Narratologie

Analyse et théorie narratives

$13 \mid 2006$

Nouvelles approches de l'intertextualité

\title{
Les enjeux intertextuels dans La Busca et Mala hierba de Pío Baroja
}

\section{Elisabeth Delrue}

\section{(2) OpenEdition}

1 Journals

Édition électronique

URL : http://journals.openedition.org/narratologie/326

DOI : 10.4000/narratologie.326

ISSN : 1765-307X

Éditeur

LIRCES

\section{Référence électronique}

Elisabeth Delrue, «Les enjeux intertextuels dans La Busca et Mala hierba de Pío Baroja », Cahiers de Narratologie [En ligne], 13 | 2006, mis en ligne le 01 septembre 2006, consulté le 15 novembre 2019 URL : http://journals.openedition.org/narratologie/326 ; DOI : 10.4000/narratologie.326

Ce document a été généré automatiquement le 15 novembre 2019.

\section{(c) (i) (9)}

Cahiers de Narratologie - Analyse et théorie narratives est mis à disposition selon les termes de la licence Creative Commons Attribution - Pas d'Utilisation Commerciale - Pas de Modification 4.0 International. 


\title{
Les enjeux intertextuels dans $L a$ Busca et Mala hierba de Pío Baroja
}

\author{
Elisabeth Delrue
}

1 L'intertextualité est un concept forgé par Julia Kristeva, dans un article de 1967, «Bakhtine, le mot, le dialogue et le roman "1, article repris en 1969 dans Séméiotiké, où elle écrit :

\begin{abstract}
« Le mot (le texte) est un croisement de mots (de textes) où on lit au moins un autre mot (texte) (...) Tout texte se construit comme mosaïque de citations, tout texte est absorption et transformation d'un autre texte. A la place de la notion d'intersubjectivité s'installe celle d'intertextualité, et le langage poétique se lit, au moins comme double. $»^{2}$
\end{abstract}

2 Le concept est ici présenté comme emprunté à la théorie littéraire de Bakhtine, alors que ce dernier emploie apparemment dans le même sens le terme dialogisme pour poser que tout texte se construit explicitement ou non, à travers la reprise d'autres textes.

3 Plus tard, Gérard Genette explore un champ que, dans Palimpsestes, il a appelé rétrospectivement la "transtextualité », et défini comme «tout ce qui met (un texte) en relation, manifeste ou secrète avec d'autres textes $»^{3}$, concept qui, autrement dit, correspond à ce qu'on désignait jusqu'alors, à la suite de Julia Kristeva, par le terme d'« intertextualité ", même s'il est beaucoup plus vaste. Pour Genette, en effet, cette «transtextualité » équivaut à la « littérarité » (l'essence des objets littéraires) ou à la " poéticité » que s'étaient ingéniés à cerner les Formalistes russes et les poéticiens des années 60 en tant qu'appartenance du texte à cet ensemble de textes qu'est la littérature, ou comme les rapports qu'il entretient avec les textes qui l'entourent et avec les genres qui l'incluent.

4 Au début de Palimpsestes, Genette énumère les cinq types de renvois intertextuels qu'il rassemble sous le terme générique de «transtextualité »: l'intertextualité au sens restreint de présence effective d'un texte dans un autre, sous les formes de la citation, du plagiat et de l'allusion, la paratextualité, ou les relations entre le texte d'escorte (titre, couverture, préface, épigraphes) et le texte proprement dit, la métatextualité qui 
renvoie aux relations de commentaire unissant un texte à un autre qui parle de lui, l'hypertextualité, définie comme "toute relation unissant un texte B à un texte antérieur A sur lequel il se greffe d'une manière qui n'est pas celle du commentaire $»^{4}$, l'architextualité, qui désigne les relations du texte avec les autres textes du même genre littéraire.

5 Michael Riffaterre, enfin, dans «La trace de l'intertexte " distingue «l'intertextualité obligatoire » et «l'intertextualité aléatoire ». La première réside, selon lui, dans des aberrations lexicales, syntaxiques ou sémantiques ne pouvant être résolues ni dans le contexte de la langue ni dans celui de l'œuvre mais seulement à la lumière d'un autre texte. La seconde est un effet de lecture qui varie en fonction de la compétence culturelle de chaque lecteur.

6 Nous prendrons, ici, l'intertextualité, au sens large de perception par le lecteur de rapports entre une œuvre et d'autres qui l'ont précédée et restreint d'« intertextualité aléatoire ", afin d'analyser les modalités du jeu intertextuel et leurs implications idéologiques et esthétiques dans les deux romans considérés dans notre étude : La Busca et Mala hierba de Pío Baroja. Ces deux livres ne présentent pas, en effet, ces aberrations ou incompatibilités apparentes relevant de l'« intertextualité obligatoire», et peuvent se lire parfaitement sans référence à un ailleurs. Leur dimension intertextuelle est plutôt aléatoire et diffuse et s'impose par une impression de «déjà lu » dans le choix des motifs narratifs, la caractérisation des personnages, les espaces privilégiés, la description, le style. L'intertextualité est le fait de l'instance narratrice, Baroja pratique le pastiche et la parodie, au sens genettien de ces termes ${ }^{6}$, de techniques narratives, mêlant dans un même récit des conventions et des motifs narratifs, des codes langagiers appartenant à des phases différentes de l'histoire littéraire et à des registres divers : le roman picaresque ${ }^{7}$, le roman-feuilleton romantique ${ }^{8}$, le réalisme, le roman à énigme, le roman d'apprentissage (Bildungsroman). C'est ce que nous allons voir à présent.

7 La reprise des hypotextes selon la modalité de l'imitation peut, à la fois, être stylistique ou se rattacher à toutes sortes d'homologies. Antonio Risco dans «Estructura de una novela picaresca, $L a$ Busca $»^{9}$ dégageait déjà les traits picaresques de ce roman. A notre avis, ils informent les deux livres : la nécessité de subvenir à ses besoins dans un monde hostile et dur comme seule préoccupation du héros Manuel Alcázar, issu, par surcroît d'une couche pauvre de la société ${ }^{10}$, son errance d'individu marginalisé en butte à la répression sociale ${ }^{11}$ ou son vagabondage de maîtres en maîtres ${ }^{12}$, le récit linéaire, enfin, qui suit les étapes successives de cet itinéraire. Pour ce qui est de la forme autobiographique de la narration, nous la retrouvons dans les histoires de vie intercalées ici ou là dans la trame, prises en charge par un narrateur à la première personne, rassemblant les traits du gueux à la moralité douteuse, paresseux, voleur ou vivant d'expédients, dépourvu d'idéal. C'est le cas de Vidal dans Mala hierba, au premier chapitre de la dernière partie et celui des prostituées la Flora et Petra la Aragonesa, quelques pages plus loin, au chapitre III. La Sociedad de los Tres que composent Manuel, Vidal et El Bizco n'est pas sans rappeler la confrérie de voleurs, de prostituées et de ruffians sur laquelle règne Monipodio dans la Séville de Rinconete y Cortadillo, autres personnages juvéniles à relier à Manuel et son jeune cousin, devenus experts dans l'art de tricher, ou la contre-société parallèle de la marginalité, avec ses propres lois et sa hiérarchie, el Bizco, Vidal et ses chefs «El Maestro », «El Cojo » et «El Intérprete ». L'onomastique, enfin, n'est pas de reste. Dans Mala hierba, le prénom de Marcos 
Calatrava renvoie sans doute au héros de Vicente Espinel, le vieil écuyer Marcos de Obregón qui raconte sa vie à un ami ermite dans l'ermitage duquel il a trouvé refuge contre le mauvais temps.

8 Quant au Bildungsroman, il point dans le faisceau d'histoires d'apprentissage à variante positive ou négative qui sous-tend les deux livres. Les œuvres racontent, en effet, les trajectoires vitales de personnages qui, à partir d'un point de départ à peu près identique (Roberto, Vidal et Manuel se sont rencontrés dans les mêmes lieux de travail ou de vie privée dans une situation aussi précaire) évoluent dans des directions opposées. Manuel, le héros y découvre, à l'épreuve de l'autre, son moi et ses forces, au long d'une libre succession d'épisodes.

9 L'imitation stylistique transparaît, quant à elle, dans la rhétorique qui reprend le manichéisme, le mélodramatisme et les stéréotypes langagiers du roman-feuilleton romantique et dans les tics d'écriture propres à l'école du réalisme. Dans le premier cas, les comparaisons éculées ${ }^{13}$, le ton larmoyant, l'esthétique du pathétique qui par des procédés stylistiques (amplification, antithèses ${ }^{14}$, hyperboles ${ }^{15}$ ) met en scène la douleur, la pitié, l'horreur, la terreur, la démesure, les expressions pléonastiques et synonymiques ${ }^{16}$, enfin, qui traduisent une volonté d'emphase, tout cela est ressenti par le lecteur comme une enflure du discours et désamorce une représentation tragique de l'existence transformée en tragi-comédie. Il suffit pour s'en convaincre de se reporter à quatre épisodes : celui de Leandro et Milagros ( $L a$ Busca, deuxième partie, chapitres II, V, VII, VIII, IX), celui de Manuel et Justa (La Busca, première partie, chapitre IV, Mala hierba, troisième partie, chapitre III), celui de Bernardo Santin et Esther (Mala hierba, première partie, chapitre II, deuxième partie, chapitre IV), celui, enfin, de Vidal et $E l$ Bizco (Mala hierba, seconde partie, chapitre IX, troisième partie, chapitre IV).

Le manichéisme, quant à lui, transparaît dans la collection exhaustive de traits pertinents, reliant aspect physique et caractère et destinée à révéler rapidement et immédiatement, l'essentiel d'une personnalité. Baroja grève ses personnages maléfiques d'un signe globalement négatif, forçant le trait jusqu'à la caricature, dans des portraits où le lexique oriente de façon univoque une évaluation qui invite à la condamnation ou à la répulsion. Souvent l'animalisation est à l'œuvre. Dans La Busca, par exemple, au chapitre I de la deuxième partie, El Bizco est décrit ainsi à travers le regard de Manuel :

«La cara del Bizco producía el interés de un bicharraco extraño o de un tic patológico. La frente estrecha, la nariz roma, los labios abultados, la piel pecosa y el pelo rojo y duro, le daban el aspecto de un mandril grande y rubio. $»^{17}$

11 L'auteur s'emploie, en outre, à ridiculiser le romantisme, en parodiant ses tics stylistiques, en énumérant son cortège de conventions et de lieux communs qui enflent et poétisent le réel, dès les premières lignes de La Busca, l'adjectif « romántica », accolé à « hora » ne laissant point de doute :

«Era, pues la hora del mister io ; la hora de la gente maleante ; la hora en que el poeta piensa en la inmortalidad, rimando hijos con prolijos y amor con dolor; la hora en que la buscona sale de su cubil y el jugador entra en él; la hora de las aventuras que se buscan y nunca se encuentran; la hora, en fin, de los sueños de la casta doncella y de los reumatismos del venerable anciano. Y mientras se deslizaba esta hora romántica, cesaban en la calle los gritos, las canciones, las riñas.(...) $»^{18}$

12 Pour ce qui est des caractéristiques formelles réalistes, Colette Becker dans Lire le Réalisme et le Naturalisme ${ }^{19}$ rappelle que la création, dans les productions de ce genre, repose sur toutes sortes de vérifications et d'informations visant à authentifier leur 
démarche et lui donner du sérieux et que la voix de l'auteur doit sembler la plus neutre possible. Ici, la recherche de documentation fait l'objet de caricature, à plusieurs reprises tout comme l'exigence d'impartialité :

«El autor no puede decirlo, y lo siente. (...) y es verdaderamente poco científico el no poder precisar con seguridad en qué momento empieza el cañamazo de este libro. $»^{20}$

«Sería el autor demasiado audaz si tratase de demostrar la necesidad matemática en que se encontraba la casa de doña Casiana de hallarse colocada en la calle de Mesoneros Romanos, antes del olivo, porque indudablemente, con la misma razón podía haber estado emplazada en la del Desengaño, en la de Tudescos, o en otra cualquiera ; pero los deberes del autor, sus deberes de cronista imparcial y verídico, le obligan a decir la verdad, y la verdad es que la casa estaba en la calle de Mesoneros Romanos, antes del Olivo. $»^{21}$

«El autor no llegó a conocer los inquilinos que habitaban los pisos altos ; (...). Por

esta causa el autor no se remonta a las alturas y se detiene en el piso principal. ${ }^{22}$

L'auteur s'amuse aussi à subvertir les recettes les plus éculées du roman-feuilleton, du roman réaliste, et du roman à énigme en en proposant une image déformée qui fait saillir leurs thèmes récurrents et les fait voir sous un jour ridicule.

14 Publié dans Le Journal des Débats de juin 1842 à octobre 1843, Les Mystères de Paris d'Eugène Sue dont le titre, d'ailleurs, sera cité dans le troisième tome de la trilogie $L a$ lucha por la vida, dont nos romans constituent les deux premiers recueils, comme lectures du protagoniste Juan, met en scène les marginaux de Paris, pauvres, petit peuple, ouvriers, bandits avec leur argot, leurs mœurs et leurs destins. Le héros de $L a$ Busca et de Mala hierba se promène au milieu des basses classes travailleuses madrilènes et de la petite bourgeoisie, ruffians, prostituées et autres malfrats qui, souvent, utilisent un langage propre. En outre, Rodolphe, le protagoniste du roman français s'aventure dans les tavernes les plus crapuleuses sauve une jeune prostituée Fleur de Marie des mains d'un ancien forçat, Le Chourineur (de l'argot, « chouriner », donner un coup de couteau), stéréotype chargé de dénoncer une justice de classe qui ne fait pas de différences entre un naturel honnête et la situation misérable qui conduit les individus au crime. Dans les deux livres espagnols, certes, les personnages fréquentent des tavernes, (La Blasa), la Flora est une prostituée qui évolue dans l'entourage du héros, el Bizco tuera Vidal à coups de couteau et dans Aurora roja, sera réhabilité par le narrateur qui le dépeint comme une des nombreuses victimes de la société injuste, Manuel, enfin, tâchera d'arracher la Justa à la prostitution. Mais, surtout, la parodie ici s'attache à démystifier les héros populaires. Contrairement à Rodolphe, le prince souverain de Gérolstein, Manuel est un personnage aboulique, velléitaire, promené d'espaces en espaces, selon les offres proposées et les congédiements successifs, dont chaque tentative d'entreprendre une vie nouvelle tourne court à la moindre occasion, un antihéros, en somme.

Quant aux thèmes du roman réaliste, on retrouve ici la cohorte d'êtres accablés par l'adversité ou la maladie, incapables de lutter, victimes de bourgeois cupides et immoraux, corrompus ou ambitieux, d'escrocs, la décomposition physique et morale, le personnage de la prostituée qui permet de peindre les misères humaines, celles de l'individu, morales et physiologiques, celles de la société, enfin, puisque ces filles sont jetées sur le trottoir par les conditions de vie et de travail et les dangers de la grande ville. Les déchéances des corps sont décrites, la saleté, les puanteurs. Il suffit de relire les descriptions des lieux clos où Manuel habite ou travaille, caractérisés par les odeurs nauséabondes, les évocations du Corralón, au chapitre II de la deuxième partie de $L a$ 
Busca, ou des foules anonymes en tant que masses hébétées, assoiffées de violence et de sexe, zoomorphisées. Mais là aussi, le tragique de la souffrance et de la mort est miné, au détour d'une phrase, par des commentaires ou des comparaisons humoristiques qui empêchent le lecteur de prendre au sérieux la face sordide des choses décrites en leur conférant une dimension grotesque. Ainsi, par exemple, au chapitre II de la première partie de La Busca, le narrateur compare la concierge avec son enfant à une femme canon d'une baraque de foire, alors qu'il vient d'évoquer un cadre sordide. Quelques pages plus loin, quand Manuel sert le repas des pensionnaires, les pois chiches sont comparés à des balles par leur dureté alors qu'ils expriment la malnutrition.

D'après Lise Queffélec, dans Le roman-feuilleton français au XIX ${ }^{e}$ siècle ${ }^{23}$, le roman à énigme se constitue d'une action criminelle, en général un meurtre et d'une enquête. Le roman suit, alors, les méandres et péripéties d'une enquête menée par un personnage, policier ou privé, jusqu'à la découverte finale du criminel. Or, ici, si l'action criminelle est bien présente, au chapitre IV de la dernière partie de Mala hierba, où le lecteur assiste au meurtre de Vidal par $E l$ Bizco, et que le détective, Ortiz, possède les qualités de l'emploi, souvent répétées ${ }^{24}$, il en est une version dégradée puisqu'il est tenu en échec alors qu'il connaît le meurtrier: l'enquête, dont le lecteur a suivi les multiples phases, reste ouverte, le détective est dans l'incapacité de retrouver le coupable qu'il traque, l'auteur préfère clore son roman sur les rancœurs de Manuel, l'acolyte d'Ortiz.

17 Quelles implications esthétiques et idéologiques attribuer à ce parti pris d'imitation déformante de modèles culturels pratiqués à des époques distinctes et dans des types de littératures différentes, rassemblés dans l'espace synchronique de nos deux romans? En premier lieu, les auteurs du début du $\mathrm{XX}^{\mathrm{e}}$ siècle dont Baroja fait partie rejettent l'esthétique romanesque en usage et souhaitent une rénovation littéraire. La création romanesque est donc en train de vivre une crise, on ne sait plus très bien comment écrire des romans. D'où la nécessité pour Baroja d'avoir recours à des modèles antérieurs que la culture propose comme réponse à la crise de l'inspiration, de l'invention, de la création. La littérature se nourrit de ses propres formes car elle ne peut se renouveler en trouvant d'autres formes d'expression du réel et l'écrivain, en quête d'un nouveau roman, pratique une écriture de l'intégration, de la réconciliation entre les solutions déjà inventées: Baroja prétend, semble-t-il, faire du nouveau en récusant les modèles anciens ou en adaptant le legs culturel qu'il s'est réapproprié.

Le regard du texte sur un autre texte est, par surcroît, une façon oblique de commenter son propre fonctionnement. L'intertextualité participe alors d'une stratégie de la distanciation par rapport aux personnages et au narrateur. Elle rappelle que les personnages sont des êtres de papier. Dans La Busca, en particulier, à plusieurs reprises, l'auteur impliqué parle de son roman à mesure qu'il se fait et commente ironiquement sa propre activité d'écrivain, ce qui entraîne la dénudation du caractère fictionnel de l'œuvre :

«En éste y otros párrafos de la misma calaña tenía yo alguna esperanza, porque daban a mi novela cierto aspecto fantasmagórico y misterioso ; (...). Yo resignado, he suprimido esos párrafos, por los cuales esperaba llegar algún día a la Academia Española, y sigo con mi cuento en un lenguaje más chabacano. ${ }^{25}$

Enfin, cette pratique délibérée de l'anachronisme favorise aussi la distanciation par l'incongruité qu'elle introduit et établit un rapport de connivence entre l'instance narratrice et son narrataire. Le lecteur est invité à un jeu de décodage qui nécessite une compétence culturelle pour identifier les éléments du texte pouvant donner lieu à une 
lecture intertextuelle. C'est sur la base d'une culture partagée que s'établit la connivence.

En outre, la relation intertextuelle pose une nature transhistorique des maux qui affectent toute société, en associant des codes littéraires historiques liés à des sociétés très différentes (l'Espagne des XVI ${ }^{e}$ et XVII ${ }^{e}$ siècles, la France du XIX $\left.{ }^{e} . ..\right)$ et confirme la dimension mythique du récit. Le collage intertextuel, en effet, évacue la diachronie, en éliminant passé et futur de la perspective temporelle, ne laissant qu'un présent inactuel, pure répétition. Le roman picaresque naît et se développe avec les hantises suscitées par la crise économique des $\mathrm{XVI}^{\mathrm{e}}$ et $\mathrm{XVII}{ }^{\mathrm{e}}$ siècles qui dépeuple les campagnes, multiplie les vagabonds, le refus du travail, perçu comme dégradant, l'émergence d'une mentalité bourgeoise et d'un début de capitalisme. Les deux romans de Baroja, publiés en 1904, sont liés à ce même type de contexte à l'aube du XX $\mathrm{X}^{\mathrm{e}}$ siècle, marqué par la corruption des mœurs politiques, le marasme que fait peser la politique officielle sur la vie nationale, les contrecoups économiques du désastre colonial, avec la situation financière catastrophique dont les répercussions se firent sentir dans le domaine social, l'abattement général et la mobilisation générale.

\section{NOTES}

1. Julia Kristeva, «Bakhtine, le mot, le dialogue et le roman » in Critique, t. XXXIII, $n^{\circ} 239$ (avril 1967), p. 438-465

2. Julia Kristeva, Séméiotikè, Paris, Ed. du Seuil, coll. Points, 1969, p. 84-85

3. Gérard Genette, Palimpsestes. La littérature au second degré, Seuil, 1982, p. 7.

4. Op. cit., p. 11-12 Genette appelle le texte B « hypertexte » et le texte A « hypotexte ».

5. Michael Riffaterre, «La trace de l'intertexte », La pensée $\mathrm{n}^{\circ} 215$, oct. 1980

6. La parodie se réduit à la «transformation textuelle à fonction ludique » Palimpestes, Seuil, 1982 p. 49 ou à « la transformation ludique d'un texte singulier » (op. cit., p. 164). Le pastiche est l'imitation d'un style.

7. Le XVIIème siècle est convoqué par les personnages eux-mêmes, dans Mala hierba, au chapitre IV de la première partie, quand la niña Chucha prête à Manuel des romans en livraisons « novelones por entregas » dont l'action se déroule à cette période. Le terme pícaro (gueux) est prononcé pour désigner, dans le même livre le personnage de Bonifacio Mingote. (Mala hierba, op. cit., p. 70)

8. Ponson du Terrail, le grand feuilletoniste français de l'ère romantique qui meurt en 1871 est mentionné de façon détournée par les protagonistes eux-mêmes à travers le héros de sa longue série des Rocambole, commencée en 1857 dans La Patrie et interrompue par la mort de son auteur : «Le Patron...c'est un colosse. Tu as lu Rocambole ? Non. Vidal resta un peu interloqué ; à ses yeux, Le Patron pouvait seul être comparé à la figure de Rocambole. » (Baroja, Pío Mala hierba, ed. Caro Raggio, Madrid, 1972, p. 250). Cette allusion est déjà en soi un clin d'œil au lecteur qui, d'emblée, est invité à mettre en jeu sa compétence culturelle pour identifier les éléments du texte proprement dit pouvant donner lieu à une lecture intertextuelle. 
9. Antonio Risco, «Estructura de una novela picaresca de Baroja, La Busca », Actas Congreso Internacional de Picaresca, I, p. 865-876.

10. Le thème de la faim commande les champs lexicaux, la mise en scène de conduites alimentaires dans la pension de famille et dans les tavernes. La nécessité impérieuse de se nourrir pousse Manuel de soupes populaires en centres de Bienfaisance. On apprend, au chapitre II de La Busca que sa mère Petra, mariée à un machiniste, vivait dans l'aisance, aux commandes d'une pension de famille et que, à la mort de celui-ci, elle dut déménager, se ruina et devint servante.

11. Les forces de l'ordre sont omniprésentes dans les deux romans, sous toutes leurs formes : les policiers, le commissariat, la prison, le tribunal. La menace policière plane sur la tête de Manuel, à chaque déplacement, lui qui, dans la dernière partie de La Busca oscille entre son lieu de travail au centre et ses espaces d'errance dans la ceinture méridionale et orientale de la ville accompagné de son cousin Vidal et de son acolyte $E l$ Bizco.

12. Dans $L a$ Busca, le protagoniste travaille successivement dans la pension de famille de Da Casiana, la cordonnerie de son oncle Sr Ignacio, une boutique de pains et légumes, puis une boulangerie. Dans Mala hierba, Manuel est au service du sculpteur Alex Monzon, de l'apprenti photographe Bernardo Santín, de Bonifacio Mingote, d'un imprimeur, et finit par travailler dans un cercle de jeux.

13. « aussi pâle qu'un cadavre ", " rapide comme l'éclair ", " ses yeux scintillaient ", « il tomba comme une masse ».

14. « un calme agité », " une douleur sereine ».

15. " il criait et vociférait », « haine terrible », " un horrible vacarme de pleurs et d'imprécations ».

16. « un artiste remarquable, supérieurement talentueux ».

17. Pío Baroja, La Busca, op. cit., p. 75 « Le visage de Louche suscitait le même intérêt qu'une bête curieuse ou qu'un tic pathologique. Son front étroit, son nez épaté, ses lèvres épaisses, ses taches de rousseur, sa chevelure rousse et rêche le faisaient ressembler à un grand mandrill doré ».

18. Pío Baroja, La Busca, Ed Caro Raggio, Madrid, 1972, p. 8 «C'était donc l'heure du mystère ; l'heure des gens inquiétants, l'heure où le poète pense à l'immortalité, en faisant rimer âme avec flamme et coeur avec douleur, l'heure où la prostituée sort de son repaire et où le joueur y entre, l'heure des aventures qu'on cherche et qu'on ne trouve jamais, l'heure, enfin, des rêves de la chaste jeune fille et des rhumatismes du vénérable vieillard. Et, tandis que se glissait cette heure romantique, dans la rue cessaient les cris, les chansons, les disputes. (...)»

19. Colette Becker, Lire le Réalisme et le Naturalisme, Dunod, Paris, 1992.

20. Pío Baroja, La Busca, op. cit, p. 8 « L'auteur ne peut le dire, et il le regrette (...)et il est vraiment peu scientifique de ne pas pouvoir préciser avec certitude à quel moment commence le canevas de ce livre ".

21. Ibidem, pp. 15-16 « L'auteur serait trop audacieux s'il essayait de démontrer la nécessité mathématique pour laquelle la maison de doña Casiana se trouvait placée dans la rue de Mesoneros Romanos, autrefois de l'olivier, car elle aurait aussi bien pu se trouver située dans celle du Désenchantement, dans celle de Tudescos, ou dans n'importe quelle autre, mais les devoirs de l'auteur, ses devoirs de chroniqueur impartial et véridique, l'obligent à dire la vérité, et la vérité est que la maison se trouvait dans la rue de Mesonero Romanos, autrefois de l'olivier ». 
22. Ibidem, p. 17 «L'auteur ne parvint jamais à connaître les locataires des étages du haut (...). C'est pourquoi l'auteur ne va pas jusqu'à ces hauteurs et s'arrête au premier étage $»$.

23. Lise Queffelec, Le roman-feuilleton français au XIXème siècle, coll. « Que sais-je », PUF, Paris 1989.

24. «Le dit Ortiz, traqueur renommé de fripouilles et de brigands (...) » (Mala hierba, op. cit, p. 306)/ «Ortiz était un flic passionné par son métier comme son père, et son instinct de chasseur était aussi affûté que celui d'un chien de chasse » (Mala hierba, op. cit., p. 311).

25. Pío Baroja, La Busca, op. cit., pp. 59-60 « Je fondais quelque espoir sur ce paragraphe et quelques autres du même style, parce qu'ils donnaient à mon roman un certain aspect fantasmagorique et mystérieux ; (...). Moi résigné, j’ai supprimé ces paragraphes par lesquels j'espérais arriver un jour à l'Académie espagnole, et je continue mon récit dans un style plus terre à terre ».

\section{RÉSUMÉS}

Nous prendrons, ici, l'intertextualité, au sens large de perception par le lecteur de rapports entre une œuvre et d'autres qui l'ont précédée et restreint d'«intertextualité aléatoire » dans la terminologie de Michael Riffaterre, afin d'analyser les modalités du jeu intertextuel et leurs implications idéologiques et esthétiques dans les deux romans considérés dans notre étude : La Busca et Mala hierba de Pío Baroja.

L'auteur pratique le pastiche et la parodie, au sens genettien de ces termes, de techniques narratives, mêlant dans un même récit des conventions et des motifs narratifs, des codes langagiers appartenant à des phases différentes de l'histoire littéraire et à des registres divers : le roman picaresque, le roman-feuilleton romantique, le réalisme, le roman à énigme, le roman d'apprentissage.

En associant dans le collage intertextuel des solutions narratives pratiquées à des époques différentes ou dans des types de littérature différentes, Baroja, en quête d'un nouveau roman, se réapproprie le legs culturel qu'il récuse parfois pour rénover l'écriture de son temps et l'instance narratrice évacue toute diachronie et pose une nature transhistorique des maux décrits.

\section{AUTEUR}

\section{ELISABETH DELRUE}

Université d'Amiens 